\title{
Minimally Invasive Technique of Masking Nonpitted Fluorosis on Young Permanent Incisors: A Clinical Trial
}

\author{
Shikha Dogra ${ }^{1}$, Virinder Goyal ${ }^{2}$, Neeru Singh ${ }^{3}$, Meenu Bhola ${ }^{4}$, Anil Gupta ${ }^{5}$, Shalini Garg ${ }^{6}$
}

\begin{abstract}
Aim: Analysis of masking potential of the resin infiltration technique with dental milestones guaranteed caries infiltration concept (DMG ICON) on nonpitted white spot lesions due to fluorosis in newly erupted permanent maxillary incisors for better esthetics and psychological wellbeing in children.

Materials and methods: This prospective interventional study was conducted on 60 newly erupted maxillary central incisors with mild, nonpitted white spot lesions of fluorosis till grade IV of the Thylstrup and Fejerskov (TF) index. The resin infiltration technique with DMG ICON (DMG, Hamburg, Germany) was used to mask lesions along with the analysis for color change using Euclidean distance, i.e., the $\Delta E$ (Delta $E$ ) unit of the CIE $L^{*} a^{*} b^{*}$ color space formula where $\Delta E=\left(\Delta L^{2}+\Delta a^{2}+\Delta b^{2}\right)^{1 / 2}$ using the image-analyzing software. Data were analyzed statistically by the SPSS software.

Results: Fifty-one tooth samples (85\%) showed complete masking of white spot lesions of fluorosis postoperatively to resin infiltration with net $\Delta E$ values less than or equal to 3.7 whereas lesions in nine tooth samples (15\%) were not masked completely with net $\Delta E>3.7$. Statistically highly significant results were obtained with the Wilcoxon signed-ranks test ( $p$ value $<0.001$ ).

Conclusion: The minimally invasive resin infiltration technique using DMG ICON is highly efficient and satisfactory for masking of nonpitted white spot lesions of dental fluorosis in newly erupted permanent central incisors as per the analysis for color change using the image-analyzing software.

Clinical significance: The outcome of masked and blended white spot lesions of fluorosis with the sound enamel in the smile zone with the resin infiltration technique was found to be a child-friendly, noninvasive, single-sitting approach with stabilized results in follow-up visits.

Keywords: Dental fluorosis, DMG ICON, Resin infiltration, White spot lesion.

World Journal of Dentistry (2020): 10.5005/jp-journals-10015-1702
\end{abstract}

\section{INTRODUCTION}

White spot lesions due to dental fluorosis pose serious esthetic problem in newly erupted permanent incisors. As a child approaches early adolescence, there is an alteration in the attitude and self-perception under the influence of peer pressure. This affects their emotional stability and self-confidence. Hence, this age group is more concerned about esthetics and they seek quick intervention, which should be permanent and less invasive. Also the awareness of modern Indian parents toward dental esthetics is increasing and they demand natural appearance of teeth with tooth-colored restorations for their children. ${ }^{1-3}$ The resin infiltration concept supports the concept of preventive dentistry by restoring the tooth in a least invasive way for regaining healthy, functional, and esthetic smiles. ${ }^{4}$ The present study was conducted in high fluoride belt area of state Punjab in India, with the fluoride content in drinking water $>1.5$ ppm. ${ }^{5}$

Dental fluorosis, if nonpitted, appears clinically as initial white spot lesion areas. This may range from narrow to pronounced opaque white lines following perikymata to irregularly shaped chalky white to cloudy opacities over the tooth surface. This appearance till grade IV of the Thylstrup Fejerskov index of dental fluorosis occurs due to change in the refractive index of the subsurface hypomineralized porous enamel with respect to the intact hypermineralized surface layer. ${ }^{6,7}$ Various minimally invasive suggested treatment modalities for esthetic treatment of such defects are microabrasion, tooth whitening procedures with bleaching agents, along with invasive procedures such as composite restorations and veneers. Microabrasion and composite veneering
1,5,6 Department of Pediatric and Preventive Dentistry, Faculty of Dental Sciences, SGT University, Gurugram, Haryana, India

${ }^{2}$ Department of Pediatric and Preventive Dentistry, Surendera Dental College and Research Institute, Sri Ganganagar, Rajasthan, India

${ }^{3}$ Department of Pediatric and Preventive Dentistry, Faculty of Dental Sciences, Desh Bhagat University, Mandi Gobindgarh, Punjab, India

${ }^{4}$ Department of Pedodontics and Preventive Dentistry, Dasmesh Institute of Research and Dental Sciences, Faridkot, Punjab, India

Corresponding Author: Shikha Dogra, Department of Pediatric and Preventive dentistry, Faculty of Dental Sciences, SGT University, Gurugram, Haryana, India, Phone:+91 9205716200, e-mail:mdsshikha87@gmail.com

How to cite this article: Dogra S, Goyal V, Singh N, et al. Minimally Invasive Technique of Masking Nonpitted Fluorosis on Young Permanent Incisors: A Clinical Trial. World J Dent 2020;11(1):41-46.

Source of support: Nil

Conflict of interest: None

procedures require removal of the surface layer of the enamel. Also patients with composite veneers always report either with dislodgement of the composite layer or its discoloration with passage of time. Further, microabrasion and bleaching procedures pose problems of postoperative sensitivity, long-term follow-up, and young patient compliance. ${ }^{8,9}$

Combating all these problems, the resin infiltration concept was introduced in the literature as therapeutic treatment of white spot lesions due to incipient caries and postorthodontic decalcifications. This is claimed to be a young patient-friendly, minimally invasive, 
definitive, and corrective therapy with a low-viscosity resin having a high penetration coefficient for nonpitted white spot lesions in newly erupted permanent incisors in young children. It works by occluding subsurface microporosities, thereby reversing healthy enamel color and translucency. ${ }^{8-10}$

Hence, the present study was planned with the aim to analyze the masking potential of resin infiltration with dental milestones guaranteed caries infiltration concept (DMG ICON) on nonpitted white spot lesions due to fluorosis in the esthetic zone with the goal of improving self-confidence and psychological development in these children.

\section{Materials and Methods}

This clinical controlled prospective interventional study was conducted in the Department of Pediatrics and Preventive Dentistry, Dasmesh Institute of Research and Dental Sciences, Faridkot, Punjab, India (district Faridkot of state Punjab, which is an endemic area of fluoride in northern India), with the fluoride content in drinking water $>1.5 \mathrm{ppm}^{5}$

\section{Sample Selection}

In this study, about 200 children with dental fluorosis between 6 years to 12 years of age reporting in the routine dental OPD with chief complaint of unesthetic white spots on newly erupted teeth were screened over a period of 2 years (from November 2012 to November 2014). Afterward, the present study was conducted on a convenient sample of 40 patients with nonpitted white spot lesions in the esthetic zone of the anterior maxillary region as per the inclusion and exclusion criteria explained below. Among these children, 60 tooth samples of 30 patients were subjected for resin infiltration with DMG ICON to analyze its masking effect postoperatively. Ethical clearance was obtained from the institutional ethical committee and an informed written consent was sought from the parents of the patients for their enrolment in the study.

\section{Inclusion Criteria}

Maxillary anterior tooth samples with mild, nonpitted white spot lesions of fluorosis till grade IV of the Thylstrup and Fejerskov (TF) index. Selected samples of fluorosed teeth were differentiated from nonfluoridic opacities by the Russell's criteria., ${ }^{711}$

\section{Exclusion Criteria}

Children who were physically or mentally challenged, with severe systemic disease, non fluoridic opacities as per the Russell's criteria, past dental history for esthetic intervention done for dental fluorosis, or allergy to dental materials or restorations. ${ }^{710,11}$

\section{Standardized Photographic Technique}

Photographs were taken with the Canon 550-D camera [(SP AF90 mm F 2.8 Di 1:1 Macrolens; Tamron, Saitama, Japan) and flash (MT 24-EX twinlight; Canon)]. The camera settings were done at a shutter speed of 1/200, F29, ISO 400 , and auto white balance. ${ }^{8}$ To avoid interference with the external source of light, all photographs were taken in a darkroom and the intensity of light was maintained constant using a photometer. The patient was asked to sit on a dental chair and each time the photograph was taken, the distance and angulation of the camera from the patient was kept constant.

\section{Operative Procedure, i.e., Resin Infiltration with DMG ICON}

Pretreatment digital photographs were taken as per described standardized conditions after the selected tooth sample was isolated using a rubber dam (for $\Delta E_{1}$ values to be taken as control) and retracted with a waxed dental floss, i.e., without clamp (Figs $1 \mathrm{~A}$, $2 \mathrm{~A}, 3 \mathrm{~A}, 4 \mathrm{~A}) .{ }^{12-14}$ This was followed by resin infiltration with DMG ICON (DMG, Hamburg, Germany) as per manufacturer's instructions.

The tooth surface was cleaned with prophylactic paste and rubber cup. Icon etch containing $15 \%$ hydrochloric acid $(15 \% \mathrm{HCl})$ was applied to erode the surface layer of the tooth and left for 2 minutes followed by rinsing and air-drying for 30 seconds. Then, Icon dry containing $99 \%$ ethanol was applied for 30 seconds followed by air-drying. This was followed by application of Icon infiltrant for 3 minutes to allow its penetration into the lesion area to maximum depth. Excess of Icon infiltrant was removed by cotton roll and dental floss from the proximal area followed by light-curing ( $3 \mathrm{M}$ ESPE, St. Paul, Minnesota) for 40 seconds. Icon infiltrant application was again repeated for 1 minute and light-cured for 40 seconds. The finally treated tooth surface was polished using polishing discs. ${ }^{8,9}$

Immediate posttreatment digital photographs were taken as per the described standardized conditions (Fig. 1B, 2B, 3B, 4B).

\section{Record and Data Analysis of Color Change Postoperatively}

Pretreatment and posttreatment photographs on the day of application were transferred in Adobe Photoshop Software 7.0 (Adobe Systems, San Jose, California) in the computer. $L^{*} a^{*} b^{*}$ coordinators of color were recorded using the grid in this software for both lesion area and the sound enamel in each tooth sample preoperatively and postoperatively. ${ }^{8,15}$

This was followed by analysis for color change using Euclidean distance, i.e., the $\triangle E$ (Delta $E$ ) unit of $C I E L^{*} a^{*} b^{*}$ color space formula, i.e., $\Delta E=\left(\Delta L^{2}+\Delta a^{2}+\Delta b^{2}\right)^{1 / 2}$. The $\Delta E$ unit quantifies minimal noticeable color change to the naked eye by calculation of the sum of differences in $L^{*}$ (lightness/darkness), $a^{*}$ (green-red), and $b^{*}$ (blue-yellow) coordinators in the above-described equation. ${ }^{16-18}$ In the oral cavity, under standardized conditions, net $\Delta E$ values less than or equal to 3.7 indicate complete blending of lesion area with the adjacent sound tooth enamel, i.e., complete masking of the lesion postoperatively to esthetic intervention, which is clinically not visible to the human eye. But net $\Delta E>3.7$ depicts mismatching of colors, i.e., the lesion is clinically visible hence not masked esthetically. $8,17,19$

Hence, pretreatment $\Delta E$ values (i.e., $\Delta E_{1}$ values, i.e., control) and posttreatment $\Delta E$ values (i.e., $\Delta E_{2}$ values) between lesion area and the sound tooth enamel of 60 samples using the above equation were calculated. This data were subjected to the statistical analysis based on net $\Delta E$ values of color change for 60 study samples (net $\Delta E$ values were calculated by analyzing difference of their $\Delta E_{2}$ and $\left.\Delta E_{1}\right)$. Clinically, esthetic stability of masked tooth samples with high patient satisfaction were observed at 1-year follow-up (1-year follow-up photographs were taken as shown in Figs $1 C, 2 C, 3 C, 4 C$ ).

\section{Results}

As per the described standardized photographic technique in the methodology, both pretreatment and posttreatment photographs of each tooth sample were transferred and projected to the grid of the image-analyzing software, i.e., Adobe Photoshop Software 7.0 (Adobe Systems, San Jose, California) in the computer. 8,15

In this grid, the specific point on the divided tooth part of the sound enamel was chosen to record most accurate values of $L a^{*} b^{*}$ coordinators of tooth color, which were kept constant pre and postoperatively, whereas $L_{1} a_{1}{ }^{*} b_{1}{ }^{*}$ were designated to preoperative coordinators of tooth color for the selected site of lesion area. For 

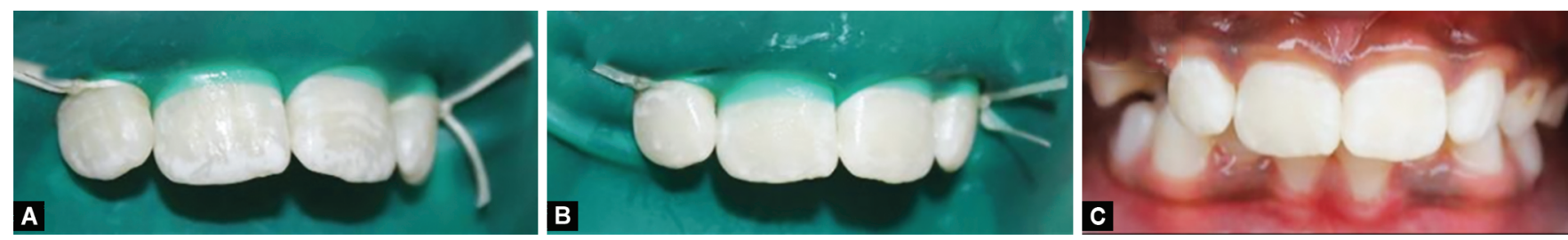

Fig. 1: Clinical photographs showing resin infiltration with DMG ICON to mask white spot lesions on permanent maxillary incisors: (A) First patient preoperative; (B) Immediate postoperative; (C) 1-year follow-up
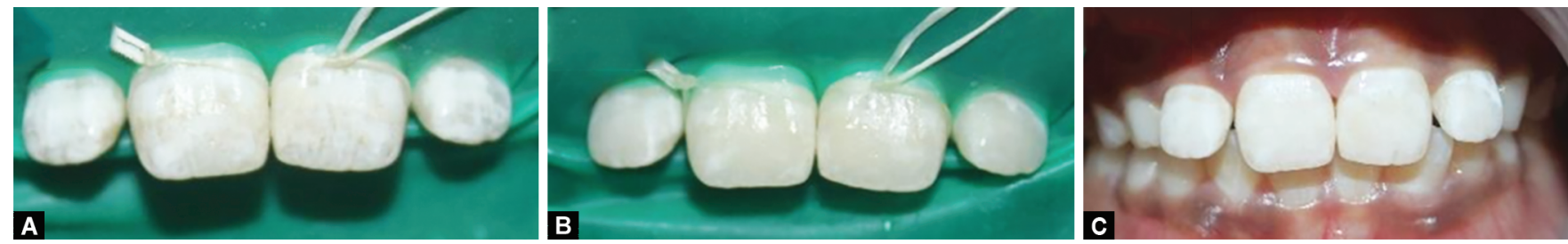

Fig. 2: Patient showing: (A) Preoperative; (B) Immediate postoperative; (C) 1-year follow-up
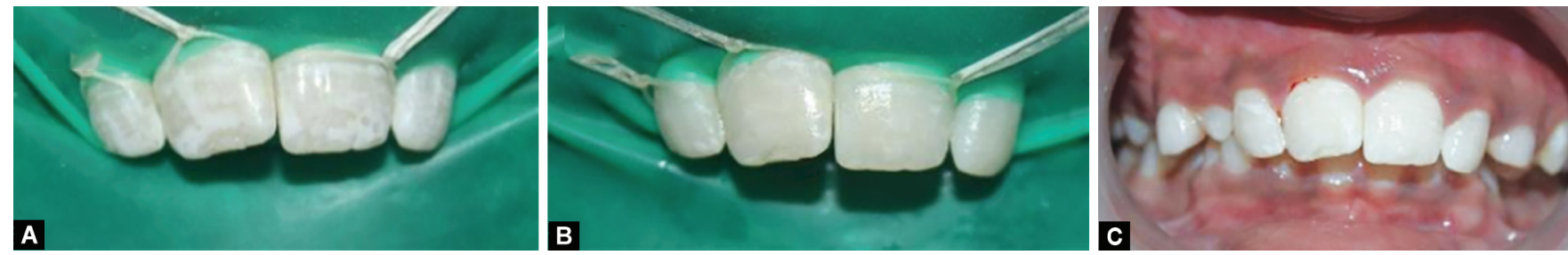

Fig. 3: Clinical photographs showing resin infiltration with DMG ICON to mask white spot lesions on permanent maxillary incisors: (A) Third patient preoperative; (B) Immediate postoperative; (C) 1-year follow-up
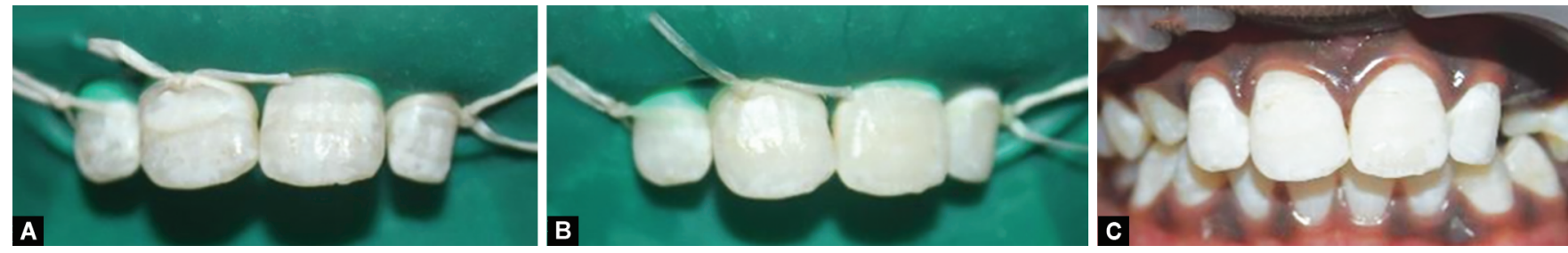

Fig. 4: Patient showing; (A) Preoperative; (B) Immediate postoperative; (C) 1-year follow-up

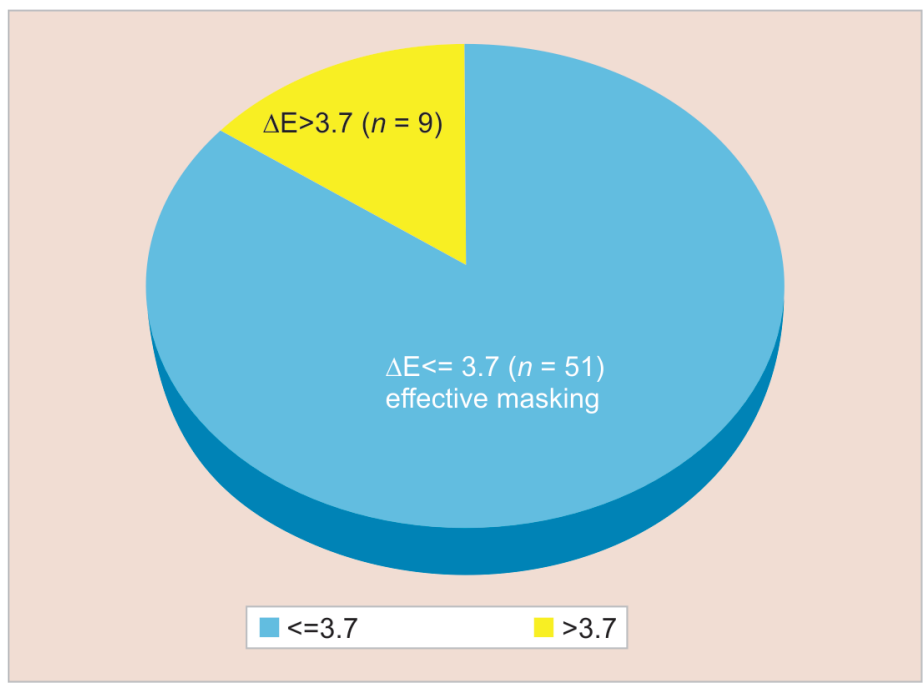

Fig. 5: Graph showing pretreatment values $\Delta E_{1}$ and posttreatment values $\Delta E_{2}$ of $n(60)$ teeth where net $\Delta E \leq 3.7$ (51) and $>3.7$ (9) 
Table 1: $\Delta E_{1}$ (pretreatment values, i.e., control), $\Delta E_{2}$ (posttreatment values), net $\Delta E$ values of $n(60)$ teeth

\begin{tabular}{|c|c|c|c|}
\hline Tooth sample & $\Delta E_{1}$ values (control) & $\Delta E_{2}$ values & Net $\Delta E$ values \\
\hline 1 & 8.12 & 5.09 & 3.03 \\
\hline 2 & 6.16 & 3 & 3.16 \\
\hline 3 & 8.66 & 8.06 & 0.6 \\
\hline 4 & 7.34 & 5.38 & 1.96 \\
\hline 5 & 12.84 & 10.67 & 2.17 \\
\hline 6 & 11.66 & 9 & 2.66 \\
\hline 7 & 13.45 & 12.84 & 0.61 \\
\hline 8 & 10.34 & 8.54 & 1.8 \\
\hline 9 & 4.13 & 3.31 & 0.82 \\
\hline 10 & 4.24 & 2.82 & 1.42 \\
\hline 11 & 5.38 & 2.23 & 3.15 \\
\hline 12 & 4.58 & 3 & 1.58 \\
\hline 13 & 8.48 & 5.19 & 3.29 \\
\hline 14 & 8.6 & 6.4 & 2.2 \\
\hline 15 & 5.19 & 4.47 & 0.72 \\
\hline 16 & 6.4 & 3.31 & 3.09 \\
\hline 17 & 10.48 & 7.07 & 3.41 \\
\hline 18 & 11.48 & 8.3 & 3.18 \\
\hline 19 & 3.46 & 3.31 & 0.15 \\
\hline 20 & 6.08 & 4.47 & 1.61 \\
\hline 21 & 6.16 & 3.31 & 2.85 \\
\hline 22 & 11.04 & 7.34 & 3.7 \\
\hline 23 & 5.91 & 5.74 & 0.17 \\
\hline 24 & 12.52 & 10.72 & 1.8 \\
\hline 25 & 9.43 & 6.7 & 2.73 \\
\hline 26 & 9.43 & 8.66 & 0.77 \\
\hline 27 & 10.24 & 7.34 & 2.9 \\
\hline 28 & 8.06 & 6.16 & 1.9 \\
\hline 29 & 12.68 & 8.18 & 4.5 \\
\hline 30 & 7.87 & 3.16 & 4.71 \\
\hline 31 & 14.49 & 11.78 & 2.7 \\
\hline 32 & 12.68 & 5.19 & 7.49 \\
\hline 33 & 9.43 & 6 & 3.43 \\
\hline 34 & 3.74 & 2.82 & 0.92 \\
\hline 35 & 6.78 & 2.82 & 3.96 \\
\hline 36 & 13.15 & 8.6 & 4.55 \\
\hline 37 & 6.4 & 6 & 0.4 \\
\hline 38 & 10.34 & 7.68 & 2.66 \\
\hline 39 & 3.6 & 3.16 & 0.44 \\
\hline 40 & 11.87 & 3.6 & 8.27 \\
\hline 41 & 6.7 & 3.6 & 3.1 \\
\hline 42 & 10.04 & 9.05 & 0.99 \\
\hline 43 & 6.16 & 3.31 & 2.85 \\
\hline 44 & 5.91 & 1.33 & 4.58 \\
\hline 45 & 9.21 & 6.08 & 3.13 \\
\hline 46 & 4.58 & 3.16 & 1.42 \\
\hline 47 & 3.74 & 3 & 0.74 \\
\hline 48 & 5.47 & 4.12 & 1.35 \\
\hline 49 & 9.43 & 6.4 & 3.03 \\
\hline 50 & 5.83 & 5.09 & 0.74 \\
\hline
\end{tabular}

\begin{tabular}{lccl} 
Contd... & \multicolumn{3}{l}{} \\
\hline Tooth sample & $\Delta E_{1}$ values (control) & $\Delta E_{2}$ values & Net $\Delta$ Evalues \\
\hline 51 & 4.35 & 3.16 & 1.19 \\
52 & 8.66 & 5.38 & 3.28 \\
53 & 5.47 & 4.89 & 0.58 \\
54 & 7.14 & 4.47 & 2.67 \\
55 & 14.17 & 11.22 & 2.95 \\
56 & 6.4 & 5 & 1.4 \\
57 & 10.81 & 3 & 7.81 \\
58 & 8.36 & 5.47 & 2.89 \\
59 & 11.91 & 6.16 & 5.75 \\
60 & 6.32 & 3.16 & 3.16 \\
\hline
\end{tabular}

each tooth sample, preoperative $\Delta E_{1}$ was calculated using the $\mathrm{CIE} L^{*} a^{*} b^{*}$ color space formula equation $\Delta E=\left(\Delta L^{2}+\Delta a^{2}+\Delta b^{2}\right)^{1 / 2}$. Preoperative coordinators of tooth color for lesion area, i.e., $L_{1} a_{1}{ }^{*} b_{1}{ }^{*}$ and calculated $\Delta E_{1}$ values (Table 1 ) of 60 tooth samples were taken as control. Similarly, after resin infiltration, postoperative coordinators of tooth color for lesion area, i.e., $L_{2} a_{2}{ }^{*} b_{2}{ }^{*}$, were obtained using the image-analyzing software and postoperative $\Delta E_{2}$ values (Table 1) were calculated using the above equation (where $L^{*} a^{*} b^{*}$ coordinators of tooth color for the sound tooth enamel were kept constant).

As shown in (Table 2), pretreatment $\Delta E_{1}$ values (control) were found in the range from 3.46 to 14.49 with mean $8.226 \pm 3.034$ and posttreatment $\Delta E_{2}$ values ranged from 1.33 to 12.84 with mean 5.641 \pm 2.644 . The net $\Delta E$ value was calculated for each tooth sample by calculating difference of postoperative $\Delta E_{2}$ and preoperative $\Delta E_{1}$ $\left(\Delta E_{2}-\Delta E_{1}\right)$ for 60 tooth samples (Table 1$)$. Postoperatively to resin infiltration, $85 \%$ study tooth samples $(n=51)$ showed complete masking of white spot lesions of fluorosis esthetically with net $\Delta E$ values less than or equal to 3.7, whereas lesions in $15 \%$ tooth samples $(n=9)$ had incomplete masking with net $\Delta E>3.7$ (i.e., net $\Delta E=3.7-5$ in $n=5$, net $\Delta E=5-7$ in $n=1$ and net $\Delta E>7$ in $n=3$ ), as shown in (Table 1 and Fig. 5), where net $\Delta E$ values were found to be in range of $0.15-8.27$ with mean $2.584 \pm 1.772$ (Table 2). These data were analyzed statistically with the Statistical Analysis Software SPSS (Statistical Package for Social Sciences by Armonk, New York) using the "Wilcoxon signed-ranks test," which showed $p$ value $<0.001$, i.e., highly significant (Table 2 ). Clinically, stability of esthetically masked lesions in these tooth samples was found to be up to patient satisfaction at 1-year follow-up (Figs 1A, 2A, 3A, 4A).

\section{Discussion}

Fluorotic white spot lesions on the labial surface of newly erupted permanent anterior teeth pose an obvious esthetic and psychological challenge in growing children. The esthetic management of these lesions without transforming the original form of the tooth could be a challenge for pediatric dentists. The perception of tooth color to the naked eye is the outcome of transmitted incident light after its absorption through mineralized dental hard tissues and subsequent scattering from the tooth surface. ${ }^{16}$ Clinical presentation of mild fluorosis as nonpitted white spot lesions on newly erupted permanent incisors occurs due to change in the refractive index of the enamel in lesion area (1.33) with respect to the sound enamel of the affected tooth (1.66). ${ }^{8,9,20}$

The resin infiltration technique with DMG ICON works on the principle of the "Chameleon effect" by occluding subsurface micropores in hypomineralized fluorosed lesions after curing of the 
Table 2: Mean values of color change (highly significant masking effect was observed postoperatively to resin infiltration with $p$ value $<0.001$ )

\begin{tabular}{lllllll}
\hline Color change & $n$ & Range & Mean $\pm S D$ & Std. error & $z$ value & $p$ value \\
\hline Preoperative $\Delta E_{1}$ & 60 & $3.46-14.49$ & $8.226 \pm 3.034$ & 0.392 & 6.736 & $<0.001^{* * *}$ \\
Postoperative $\Delta E_{2}$ & 60 & $1.33-12.84$ & $5.641 \pm 2.644$ & 0.341 & & \\
Net color change $\left(\Delta E_{2}-\Delta E_{1}\right)$ & 60 & $0.15-8.27$ & $2.584 \pm 1.772$ & 0.229 & & \\
\hline
\end{tabular}

infiltrated low-viscosity resin having a refractive index comparable to that of the enamel (1.52), which achieves required blending of the masked lesion with the sound enamel. ${ }^{8,9,20}$ Additionally, the resin infiltrate has low contact angle, high surface tension, and high coefficient of penetration to complete lesion depth favoring its flow by capillary-driven forces into subsurface microporosities leading to the required refractive index for effective masking. ${ }^{21}$

In this study, the masking of 60 tooth samples with fluoridic white spot lesions in the esthetic zone (as per the inclusion criteria) was done by the resin infiltration technique with DMG Icon. Clinically, selection of tooth samples for resin infiltration in the present study was attempted in accordance to the TF index of dental fluorosis. ${ }^{10}$ It is more sensitive than the Dean's index to provide elaborated clinical presentation of various grades of dental fluorosis (specially in endemic areas) in correlation with histological aspects of the affected enamel, further increasing its validity and reliability. ${ }^{7}$ Various studies have shown that the imageanalyzing software analyzing standardized clinical photography is more objective and reliable in quantifying lesion color and size. Even spectrophotometers and colorimeters are sensitive to measure tooth color change, but colorimeters are less accurate than spectrophotometers because they have significant edge loss, which leads to error in color measurement with variation in inter instrument reliability. ${ }^{15}$ Hence by the application of color science in dentistry, we can analyze the minimal noticeable change in tooth color using colorimeters, spectrophotometers, and imageanalyzing softwares for in vivo studies. ${ }^{15,16}$ All these techniques can evaluate minimal noticeable color change to the human eye by obtaining Euclidean distance, i.e., $\triangle E$ (Delta $\mathrm{E}$ ), from the CIELAB color space formula using equation $\Delta E=\left(\Delta L^{2}+\Delta a^{2}+\Delta b^{2}\right)^{1 / 2}$, where coordinators of physical intensity of color have perceptual meaning, i.e., $L^{*}$ (lightness/darkness), $a^{*}$ (green-red), and $b^{*}$ (blue-yellow). ${ }^{16-18}$

Keeping in mind the basis of the resin infiltration concept as a noninvasive procedure, following strict manufacturer's instructions neither we have repeated the etching step for complete removal of the surface layer for its complete erosion nor we have increased etching and application time of Icon infiltrant. Although few percentages of tooth samples (15\%) in this study showed inappropriate results postoperatively, yet $85 \%$ of study samples have shown successful masking of lesions esthetically with highly significant results statistically ( $p$ value $<0.001$ ). High patient satisfaction in terms of esthetic stability of masked lesions in these tooth samples was observed clinically at 1-year follow-up (Figs 1A, 2A, 3A, 4A), which has predicted resin infiltration as singlesitting, quick, noninvasive, painless treatment of these lesions while maintaining shape, size, form, and function of the sound tooth structure without its additional loss in an economical manner than conventional approaches without relapse of treated lesions.

DMG ICON used in present study contains $15 \% \mathrm{HCl}$ (rather than $37 \%$ ) as Icon etch to erode the surface hypermineralized layer for exposure of subsurface microporosities. Thereby, in accordance to the modern era of minimally invasive dentistry, Icon etch removes only about $40 \mu \mathrm{m}$ of the surface layer, whereas initial tooth reduction in microabrasion removes about $360 \mu \mathrm{m}$ of the surface enamel. Following this step, neither cavitation occurred nor any manual tooth reduction is required in contrast to composite restorations or veneers and microabrasion. 8,9,21,22

Further complete desiccation was achieved by application of Icon dry containing $99 \%$ ethanol for 30 seconds to remove water filled in exposed micropores of the etched surface of the lesion to facilitate the diffusion of Icon Infiltrant, i.e., the low-viscosity resin into the lesion body to occlude subsurface microporosities. ${ }^{8-10}$ In comparison to this fact, bleaching techniques treat only superficial layers of the enamel, not from inside of the complete lesion body, therefore their results are dramatic, inconsistent, and timeconsuming. ${ }^{9,23}$

In this clinical control trial prospective interventional study, 51 tooth samples (85\%) showed complete masking of white spot lesions of fluorosis postoperatively to resin infiltration with net $\Delta E$ values less than or equal to 3.7, whereas these lesions in nine tooth samples (15\%) were not masked completely with net $\Delta E>$ 3.7 (Table 1, Fig. 5), which was in accordance with the literature review of the resin infiltration technique. Auschill et al. did esthetic evaluation of mild to moderate fluorosis stains by resin infiltration with DMG ICON in 2015. After follow-up of 6 months, they found resin infiltration to be an agreeable option for these lesions than other invasive, conventional procedures but they emphasized that long-term outcomes of this technique need to be determined by further studies. ${ }^{24}$ Also Gugnani et al. analyzed esthetic changes in nonpitted fluorosis stains in 2017 by comparative postoperative effects of resin infiltration, in office bleaching and combination therapies. They reported that resin infiltration alone or in combination with bleaching therapy showed better results than bleaching therapy alone in these defects. ${ }^{10}$ In 2018, Di Giovanni et al. did a systemic review of interventions for dental fluorosis in six clinical trials and they found highest percentage of success in esthetic improvement with resin infiltration technique alone or its combination with bleaching therapy followed by bleaching alone, which was least with microabrasion. ${ }^{23}$ Theodory et al. in 2019 conducted an in vitro study to compare the masking ability and penetration capacity amongst three resin composite sealers i.e., Biscover LV (Bisco), Optiguard (Kerr Hawe), Permaseal (Ultradent and resin infiltrant i.e., DMG ICON with control group as no treatment. Procedures were done in shallow artificial caries lesions of seventy five extracted human molars. They concluded that resin infiltrant DMG ICON showed the deepest penetration of all resins on analysis of clinical photographs based on $100-\mathrm{mm}$ visual analogue scale (VAS). ${ }^{25}$ Further long-term camouflage effects of resin infiltration (Icon, DMG, Hamburg, Germany) was reassessed by Knosel et al. in an in vivo study of 20 subjects. They found that assimilation of the infiltrated white spot lesion to the color of the sound adjacent enamel by resin infiltration was suitable for the long-term improvement in the esthetic appearance of postorthodontic white spot lesions. ${ }^{26}$ Also Garg and Chavda managed three cases of mild to moderate fluorosis by combination of bleaching and the resin infiltration technique followed by quantification of color change 
using the Adobe photoshop software using the CIEDE2000 formula and they achieved satisfactory masking of these lesions, which was stable till 12 months of follow-up. ${ }^{27}$

Hence, our results advise the use of the resin infiltration technique to successfully mask white spot lesions of dental fluorosis ranging from grade I to IV of the TF index with stable esthetic masking of these lesions at 1-year follow-up. This child-friendly technique aid in developing a better psychology in growing children.

\section{Conclusion}

The minimally invasive resin Infiltration technique using DMG ICON is highly efficient in masking nonpitted white spot lesions of dental fluorosis in newly erupted permanent central incisors, which enhances the esthetics, self-confidence, and psychological well-being in children.

\section{Clinical Significance}

The outcome of masked and blended white spot lesions of fluorosis with the sound enamel in the smile zone with the resin infiltration technique was found to be a child-friendly, noninvasive, singlesitting approach with stabilized results in follow-up visits.

\section{Limitations of Study}

- Resin infiltration is a technique-sensitive procedure and an inefficient isolation may lead to complete failure of the procedure.

- The masking effect of resin infiltration does not work for deep lesions caused by fluorosis.

- The present study reports stable masking of fluorotic nonpitted lesions over a period of 2 years; further studies of longer duration are required to assess this masking effect further.

\section{Acknowledgments}

I would like to pay my regards and thanks to Dr Neeraj Gugnani (Professor, Department of Pedodontics and Preventive Dentistry, D.A.V Dental College, Yamuna Nagar, Haryana, India) for helping me in this study. Manufacturer of resin infiltrate, i.e., DMG ICON: DMG Chemisch-Pharmazeutische, Hamburg, Germany.

\section{References}

1. Kavand G, Broffitt B, Levy SM, et al. Comparison of dental esthetic perceptions of young adolescents and their parents. J Public Health Dent 2012;72(2):164-171. DOI: 10.1111/j.1752-7325.2011.00306.x.

2. Coleman JC, Hendry LB. The nature of adolescents. 3rd ed., London: Routledge; 1999.

3. Josefsson E, Lindsten R, Hallberg LR. A qualitative study of the influence of poor dental aesthetics on the lives of young adults. Acta Odontol Scand 2010;68(1):19-26. DOI: 10.3109/00016350903281740.

4. Bahadır HS, Karadag G, Bayraktar Y. Minimally invasive approach for improving anterior dental aesthetics: case report with 1-year follow-up. Case Rep Dent 2018;2018:4601795. DOI: 10.1155/2018/4601795.

5. Angura La ML, Singh BP. Ground water year book of Punjab and Chandigarh (UT). 2017. 64-65.

6. Mascarenhas AK. Risk factors for dental fluorosis: a review of the recent literature. Pediatr Dent 2000;22(4):269-277.

7. Adelario AK, Vilas-Novas LF, Castilho LS, et al. Accuracy of the simplified Thylstrup \& Fejerskov index in rural communities with endemic fluorosis. Int J Environ Res Public Health 2010;7(3):927-937. DOI: 10.3390/ijerph7030927.
8. Kim S, Kim EY, Jeong TS, et al. The evaluation of resin infiltration for masking labial enamel white spot lesions. Int J Paediatr Dent 2011;21(4):241-248. DOI: 10.1111/j.1365-263X.2011.01126.x.

9. Gugnani N, Pandit IK, Goyal V, et al. Esthetic improvement of white spot lesions and non-pitted fluorosis using resin infiltration technique: series of four clinical cases. J Indian Soc Pedod Prev Dent 2014;32(2):176. DOI: 10.4103/0970-4388.130996.

10. Gugnani N, Pandit IK, Gupta M, et al. Comparative evaluation of esthetic changes in nonpitted fluorosis stains when treated with resin infiltration, in-office bleaching, and combination therapies. J Esthet Restor Dent 2017;29(5):317-324. DOI: 10.1111/jerd.12312.

11. Jackson RD, Kelly SA, Katz B, et al. Dental fluorosis in children residing in communities with different water fluoride levels: 33-month follow-up. Pediatr Dent 1999;21(4):248-254.

12. Korkut B, Yanikoglu F, Tagtekin D. Direct midline diastema closure with composite layering technique: a one-year follow-up. Case Rep Dent2016 2016. DOI: 10.1155/2016/6810984.

13. Xia J, Li Y, Cai D, et al. Direct resin composite restoration of maxillary central incisors using a 3D-printed template: two clinical cases. BMC Oral Health 2018;18(1):158. DOI: 10.1186/s12903-018-0621-4.

14. Winters J, Cameron AC, Widmer RP. Pulp therapy for primary and immature permanent teeth. In Handbook of Pediatric Dentistry Mosby 2013. pp. 103-122.

15. Chu SJ, Trushkowsky RD, Paravina RD. Dental color matching instruments and systems. Review of clinical and research aspects. J Dent 2010;38:e2-e16. DOI: 10.1016/j.jdent.2010.07.001.

16. Joiner A, Hopkinson I, Deng $Y$, et al. A review of tooth colour and whiteness. J Dent 2008;36(Suppl 1):S2-S7. DOI: 10.1016/ j.jdent.2008.02.001.

17. Kannan A, Padmanabhan S. Comparative evaluation of icon resin infiltration and $\mathrm{Clinpro}^{\mathrm{TM}} \mathrm{XT}$ varnish on colour and fluorescence changes of white spot lesions: a randomized controlled trial. Prog Orthod 2019;20(1):23. DOI: 10.1186/s40510-019-0276-y.

18. Leland A, Akyalcin S, English JD, et al. Evaluation of staining and color changes of a resin infiltration system. Angle Orthod 2016;86(6): 900-904. DOI: 10.2319/111615-777.1.

19. Chang JY, Chen WC, Huang TK, et al. Evaluation of the accuracy and limitations of three tooth-color measuring machines. J Dent Sci 2015;10(1):16-20. DOI: 10.1016/j.jds.2013.04.004.

20. Cocco AR, Lund RG, Torre E, et al. Treatment of fluorosis spots using a resin infiltration technique: 14-month follow-up. Oper Dent 2016;41(4):357-362. DOI: 10.2341/14-335-S.

21. Paris $\mathrm{S}$, Meyer-Lueckel H. Masking of labial enamel white spot lesions by resin infiltration-a clinical report. Quintessence Int 2009;40(9): 713-718.

22. Tong LS, Pang MK, Mok NY, et al. The effects of etching, microabrasion, and bleaching on surface enamel. J Dent Res 1993;72(1): 67-71. DOI: $10.1177 / 00220345930720011001$.

23. Di Giovanni T, Eliades T, Papageorgiou SN. Interventions for dental fluorosis: a systematic review. J Esthet Restor Dent 2018;30(6): 502-508. DOI: 10.1111/jerd.12408.

24. Auschill TM, Schmidt KE, Arweiler NB. Resin infiltration for aesthetic improvement of mild to moderate fluorosis: a six-month follow-up case report. Oral Health Prev Dent 2015;13(4):317-322. DOI: 10.3290/ j.ohpd.a32785.

25. Theodory TG, Kolker JL, Vargas MA, et al. Masking and penetration ability of various sealants and ICON in artificial initial caries lesions in vitro. J Adhes Dent 2019;21(3):265-272. DOI: 10.3290/j.jad. a42520.

26. Knosel M, Eckstein A, Helms HJ. Long-term follow-up of camouflage effects following resin infiltration of post orthodontic white-spot lesions in vivo. Angle Orthod 2018;89(1):33-39. DOI: 10.2319/052118383.1.

27. Garg SA, Chavda SM. Color masking white fluorotic spots by resin infiltration and its quantitation by computerized photographic analysis: a 12-month follow-up study. Oper Dent 2020;45(1):1-9. DOI: 10.2341/17-260-T. 